\title{
Microscale recording from human motor cortex: implications for minimally invasive electrocorticographic brain-computer interfaces
}

\author{
Eric C. Leuthardt, M.D. ${ }^{1,2}$ ZAC Freudenberg, M.S., $^{3}$ David Bundy, B.S., ${ }^{1}$ \\ AND JAROD ROLAND, B.S. ${ }^{2}$ \\ Departments of ${ }^{\prime}$ Biomedical Engineering, ${ }^{2}$ Neurological Surgery, and ${ }^{3}$ Computer Science, \\ Washington University in St. Louis, Missouri
}

\begin{abstract}
Object. There is a growing interest in the use of recording from the surface of the brain, known as electrocorticography (ECoG), as a practical signal platform for brain-computer interface application. The signal has a combination of high signal quality and long-term stability that may be the ideal intermediate modality for future application. The research paradigm for studying ECoG signals uses patients requiring invasive monitoring for seizure localization. The implanted arrays span cortex areas on the order of centimeters. Currently, it is unknown what level of motor information can be discerned from small regions of human cortex with microscale ECoG recording.

Methods. In this study, a patient requiring invasive monitoring for seizure localization underwent concurrent implantation with a 16-microwire array (1-mm electrode spacing) placed over primary motor cortex. Microscale activity was recorded while the patient performed simple contra- and ipsilateral wrist movements that were monitored in parallel with electromyography. Using various statistical methods, linear and nonlinear relationships between these microcortical changes and recorded electromyography activity were defined.

Results. Small regions of primary motor cortex $(<5 \mathrm{~mm})$ carry sufficient information to separate multiple aspects of motor movements (that is, wrist flexion/extension and ipsilateral/contralateral movements).

Conclusions. These findings support the conclusion that small regions of cortex investigated by ECoG recording may provide sufficient information about motor intentions to support brain-computer interface operations in the future. Given the small scale of the cortical region required, the requisite implanted array would be minimally invasive in terms of surgical placement of the electrode array. (DOI: 10.3171/2009.4.FOCUSO980)
\end{abstract}

\section{KeY WordS - electrocorticography - brain-computer interface $\quad$ motor cortex}

$I^{2}$ $\mathrm{N}$ the past five years there has been growing interest in the use of cortical recording from the surface of the brain, known as ECoG, as a practical signal platform for BCI application. The 2 predominant preexisting modalities prior to its introduction were brain signals acquired from the scalp, known as EEG, or single-neuron recording of action potential spiking, referred to as "single units." The use of ECoG has several notable advantages over these 2 modalities with regard to signal quality and durability. Its signals are more robust and have access to the high-frequency oscillations, known as gamma rhythms, not accessible to EEG because of the low-pass filtering nature of the skull. $3,8,31$ These high frequencies

\footnotetext{
Abbreviations used in this paper: $\mathrm{BCI}=$ brain-computer interface; $\mathrm{ECoG}=$ electrocorticography; $\mathrm{EEG}=$ electroencephalography; $\mathrm{EMG}=$ electromyography.
}

have been shown to represent significant amounts of information concerning focal cortical populations of activation and representation of simple and complex motor intentions. ${ }^{17,20,28}$ Additionally, because ECoG signals are acquired from the surface of the brain and do not require intraparenchymal electrode insertion (as with single units), signal-prohibitive encapsulation (which is an obstacle in chronic intraparenchymal recording) is less likely for long-term implantation with ECoG-based systems..$^{1,32,33}$ This combination of high signal quality and long-term stability has been posited to be the ideal intermediate modality for practical clinical application in the future. ${ }^{16}$

There has been substantial progress in the exploration and development of ECoG as a signal substrate for effective neuroprosthetic application. The first use of ECoG to achieve device control was accomplished in 2004. Amplitude changes in high-frequency gamma rhythms 




FIG. 1. Experimental setup showing the microelectrode dimensions, anatomical location based on reconstructed MR and CT images of implanted electrodes, and EMG recording electrodes on the arm.

were used to allow individuals to gain rapid and effective control of a 1D cursor on a computer screen. ${ }^{17}$ Achieving control in $<30$ minutes was a substantial improvement compared with more prolonged training requirements involved with either EEG or single-unit systems., ${ }^{9,14}$ The cortically constrained nature of these high-frequency changes has allowed multiple independent motor signals to be acquired that can allow individuals to achieve 2D control of a cursor on par with invasive single-unit systems. ${ }^{30}$ Again, this multidimensional control occurred on the order of minutes versus the months to years required with other systems. ${ }^{9,35}$ Taken together, these findings support the notion that ECoG signals have sufficient information to potentially provide effective control in the future.

To date, the research paradigm for studying ECoG signals has involved patients with intractable epilepsy requiring invasive monitoring for seizure localization. This in general requires a large craniotomy for placement of an electrode array, known as "grids," often spanning 6-8 cm in maximal dimension. The spacing between electrodes is typically $1 \mathrm{~cm}$. From a functional standpoint this is anatomically coarse. Despite this typical arrangement, cortical signals have been sufficient to distinguish multidimensional hand movements. These observations were found to often come from only a few of the electrodes from the larger array. ${ }^{28}$ The spatial resolution, as it relates to independent signals in the cortex, is actually much finer on the order of millimeters. ${ }^{3,8,31}$ In some preliminary studies in humans, smaller electrode array configurations have been shown to reveal more finely resolved information about speech processing. ${ }^{2}$ Thus, for future considerations of a BCI implant, it will be important to understand what degree of cortical coverage will be required to provide sufficient information about a given motor intention that could be used for brain-derived device control.

At this point it is not known what level of motor information can be discerned from a small region of human cortex with microscale ECoG recording. We have studied a patient requiring invasive monitoring for seizure localization. Concurrent with their standard electrode arrays for monitoring purposes, a 16-contact microwire array (1-mm electrode spacing) was placed over primary mo- tor cortex. Microscale brain activity was recorded while the patient performed simple contra- and ipsilateral wrist movements that were monitored in parallel with EMG. Using various statistical methods, we defined linear and nonlinear relationships between these microcortical changes and recorded EMG activity. We find that small regions of primary cortex carry enough information to distinguish between various states of motor movement. Sufficient information was present to discern wrist flexion and extension and to effectively distinguish ipsi- and contralateral movements. These findings support the conclusion that small regions of ECoG-investigated cortex may provide sufficient information about motor intentions to support BCI surgeries in the future. Additionally, given the small scale of the cortical region required for sufficient signal, the requisite implanted array would be minimally invasive in terms of surgically placing the electrode array that likely would not exceed a bur hole.

\section{Methods}

\section{Overview}

To test the level of information present in human primary motor cortex, we evaluated a 59-year-old righthanded Caucasian woman in whom a right occipitoparietal grid was required for seizure localization. In addition to the ECoG grid array, a 16-contact microarray was placed over the primary motor cortex. Data from the microarray were recorded while the patient performed simple cue-directed wrist flexion and extension movements with either the right (ipsilateral) or the left (contralateral) wrist. Concurrent with micro-ECoG recording, muscle activity was documented with EMG electrodes placed on the ventral and dorsal surface of the right and left forearms (Fig. 1). We analyzed the micro-ECoG recordings to determine the location of significant amplitude changes in various frequency bands during the motor tasks. The results of these analyses were compared between limbs and between movements (flexion vs extension). The study was approved by the Human Research Protection Office at Washington University in St. Louis. 


\section{Microscale ECoG recording in the human motor cortex}

\section{Surgical Placement and Experimental Setup}

The 16-contact microarray was placed intraoperatively in conjunction with the standard electrodes for seizure monitoring. The microarray was placed over primary cortex that was accessible from the craniotomy for the standard grid electrode placement. Location was established using anatomical landmarks to determine the central sulcus. These landmarks were visualized with intraoperative stereotactic navigation and then confirmed on the surface of the brain. After the patient had undergone implantation, he/she was admitted to the epilepsy monitoring unit. Once there, the patient was seated 75-100 cm away from a standard flat screen monitor where visual stimuli were presented. Visual cues for movement initiation were presented using the BCI2000 program. ${ }^{29}$ The BCI2000 program is a general-purpose system for data acquisition, stimulus presentation, and brain monitoring. In the context of brain mapping, it supports programmable presentation of stimuli and simultaneous ECoG and EMG signal recordings. It associates the timing of these stimuli with the recorded physiological signals that facilitates offline analyses. The signal from the patient's subdural microarray was connected to a separate FDA-approved amplifier/digitizer system (g.tec-Guger Technologies) that was connected to an acquisition computer running BCI2000. The microarray electrodes were referenced to an inactive intracranial electrode, amplified, bandpass filtered $(0.15-500 \mathrm{~Hz})$, digitized at $1200 \mathrm{~Hz}$, and stored. The microarray consisted of 16 microwire electrodes that were $75 \mu$ in diameter and spaced $1 \mathrm{~mm}$ apart from center to center. The electrodes were arranged in an $4 \times 4$ arrangement (total of $4 \times 4-\mathrm{mm}$ cortical coverage) and embedded in Silastic plastic (total array diameter $6.35 \mathrm{~mm}$ ) (Fig. 1, Microelectrode).

\section{Stimuli and Motor Movement}

In the experimental paradigm patients received visual cues to move either the "right wrist" or "left wrist." The cue would be present on the screen for 3 seconds (a trial), and a rest interval of 1.5 seconds would be present in which the screen would be blank. The patient was instructed to continuously flex and extend his/her cued hand at the wrist when the cue was present and stop when the cue disappeared. The total session included 54 trials per hand lasting a total of 8.1 minutes. Because each trial involved several repetitions of wrist movement during the cued period, the task cueing code does not track actual wrist movements. A better measurement of actual wrist movements was achieved through the use of 2 EMG electrodes placed on the patients dorsal and ventral forearms (a total of 8 electrodes, 4 per arm) (Fig. 1).

\section{Statistical Analysis}

Statistical Difference in Time Frequency Features Between Right and Left Wrist Movement Periods. Both EEG and macro-ECoG grid recordings have been found to contain significant information about the brain state within the frequency domain. For this reason, the microECoG grid recordings were analyzed in the frequency domain using visually cued wrist movements. The ECoG signals were split into periods of 4.15 seconds after the beginning of each right and left wrist movement cue period. The frequency space was exponentially sampled from 1 to $600 \mathrm{~Hz}$, using a total of 39 frequencies; the wavelets were created by combining a complex sine/cosine waveform for each frequency with a Gaussian envelope whose (variance or standard deviation) was 3 wavelengths of the frequency. This frequency transform was computed for each movement period using a Gabor wavelet dictionary that fits each frequency in the frequency feature space with an appropriately sized wavelet. A wavelet dictionary has advantages over binned spectral transform methods such as fast Fourier transform because it yields good temporal resolution at both high and low frequencies. The convolution of each such Gabor wavelet was then computed with each channel of the ECoG signal to create a time-frequency representation (spectrogram) for each wrist movement period.

Probability values of statistical difference between the right and left wrist movement periods were then calculated for each time-frequency point using a 2-sample t-test across the 54 right and left wrist movement periods. Each $\mathrm{p}$ value was adjusted using Bonferroni corrections to account for multiple comparisons across electrodes, frequencies, and time. All t-test results presented depict features that either increased or decreased between the 2 conditions with a Bonferroni-corrected $\mathrm{p}$ value $<0.05$.

Mutual Information. Mutual information is a quantification of the information gained about a random variable $\mathrm{X}$ from the measurement of a second system Y. ${ }^{4}$ If $\mathrm{X}$ and $\mathrm{Y}$ are independent, then their mutual information is 0 . However, if knowing the value of $Y$ reduces the uncertainty of the value of $\mathrm{X}$, then the mutual information between the 2 systems is $>0$. The average amount of information gained from any measurement of $\mathrm{X}$ is the entropy $(\mathrm{H})$ of $\mathrm{X}$. Using entropy the mutual information (MI) between $\mathrm{X}$ and $\mathrm{Y}$ can be defined as follows:

$$
\begin{aligned}
& \mathrm{MI}(\mathrm{X}, \mathrm{Y})=\mathrm{H}(\mathrm{X})+ \\
& \mathrm{H}(\mathrm{X} / \mathrm{Y}),
\end{aligned}
$$

where $\mathrm{H}(\mathrm{X} / \mathrm{Y})$ is the information about the value of $\mathrm{X}$ gained as a measurement of $\mathrm{Y}$.

Formally the entropy $\mathrm{H}(\mathrm{X})$ is defined as:

$$
H(X)=\int_{-\infty}^{\infty} P_{X}(x) \log 2\left(P_{X}(x)\right)
$$

where $P_{X}(x)$ is the probability that $X=x$ in the system $X$. While it is usually not feasible to exactly define probabilities for real systems, they can be estimated as histograms. Thus, in practice when there are time series measurements, $\mathrm{X}=\left\{\mathrm{x}_{1}, \mathrm{x}_{2}, \ldots, \mathrm{x}_{\mathrm{n}}\right\}$ and $\mathrm{Y}=\left\{\mathrm{y}_{1}, \mathrm{y}_{2}, \ldots\right.$. $\left.\mathrm{y}_{\mathrm{n}}\right\}$, that can take on measurement values $\left\{\mathrm{i}_{1}, \mathrm{i}_{2}, \ldots, \mathrm{i}_{\mathrm{m}}\right\}$ and $\left\{\mathrm{j}_{1}, \mathrm{j}_{2}, \ldots, \mathrm{j}_{\mathrm{m}}\right\}$, respectively, then the mutual information between systems $\mathrm{X}$ and $\mathrm{Y}$ can be estimated as: 


$$
\begin{aligned}
& M(X, Y)= \\
& \sum_{i=1: m}^{j=1: m}\left\{\sum_{t=1: n}\left(x_{t}=i \cap y_{t}=j\right) \log _{2}\left(\sum_{t=1: n}\left(x_{t}=i \cap y_{t}=j\right) /\left(\left(\sum_{t=1: n} x_{t}=i\right) *\left(\sum_{t=1: n} y_{t}=j\right)\right)\right)\right\}
\end{aligned}
$$

Mutual information is related to covariance, but it has the advantage that the relationship between the values of $\mathrm{X}$ and $\mathrm{Y}$ does not need to be linear. Any relationship that is consistent over the duration the measurements are taken increases the mutual information score.

Mutual Information Time-Frequency Features. Breaking up the ECoG signal into segments corresponding to right and left flexion and extension and then calculating the statistically significant spectral features in a blocked format (as with right and left movement) is not ideal and loses information. This is because flexion and extension do not occur in isolation of each other, but rather occur in a continuous fashion. Therefore, it is not optimal to compute the mutual information between the spectral signals and a simple binary feature describing if the behavior is a flexion or extension. More accurate information is available from the EMG recording, which accurately tracks wrist flexion and extension as a continuous time-varying measurement. The mutual information was computed between each frequency feature of the microgrid and the 4 EMG signals. To detect time-shifts between the cortical signal and the EMG signal, mutual information was computed with the EMG signals shifts forward and backward in time by $332 \mathrm{msec}$ relative to the ECoG spectrogram.

To account for the intrinsically information-rich nature of both the ECoG and EMG signal, a threshold for mutual information was created. The mutual information value between a given EMG recorded electrode (Electrodes 1-8) and the summated signal between the 2 electrodes from a given site (right and left dorsal forearm and ventral forearm) was set as the cut-off. Because the EMG signals were calculated directly from these channels, it can be expected that these mutual information scores represent high degrees of mutual information given the limitations of the recording paradigm. For each of these comparisons, a maximum mutual information score of approximately 0.03 was found. This score was then used to scale the mutual information features of the microgrid.

\section{Anatomical Localization of Microarray}

To localize the microarray, preoperative T1-weighted structural MR imaging and postoperative CT scanning studies showing electrode placement were reconstructed using Analysis of Functional NeuroImages, ${ }^{5}$ and a cortical reconstruction and volumetric segmentation of the structural MR imaging was performed using the Freesurfer image analysis suite (http://surfer.numr.mgh.harvard.edu/). ${ }^{7} \mathrm{~A}$ local Pearson correlation was used to align the reconstructed CT and MR images. ${ }^{27}$ Due to mass effect from implanted hardware and perioperative volume changes, aligned electrode positions appeared deep to the surface of the cortex. To correct for this, a vector normal to the cortical surface was estimated, and the array was projected to the surface along this normal vector (Fig. 1).

\section{Results}

\section{Separating Contra- and Ipsilateral Movements}

The ECoG signals from the microarray electrodes that were recorded during the conditions of left (contralateral) hand movement and right (ipsilateral) hand movements were compared. Using a Gabor wavelet dictionary, the raw signal was converted to the spectral domain for each active condition and rest interval. The spectra were averaged across each trial and compared between each active condition and rest and between the 2 active conditions of right versus left movement. Each active condition was significantly different from rest and the other active condition ( $p<0.05$, Bonferroni corrected for each electrode, frequency band, and point in time). Most notably, the differences between right- and left-sided movements were distinct both in anatomical location (which microelectrode demonstrated a significant change) and frequency spectra (at what frequency there was a significant amplitude change). As shown in Fig. 2, the differences were predominantly located in lower-frequency bands $(<$ $40 \mathrm{~Hz}$ ) and in Electrodes 1, 2, 8, 9, and 10. These findings demonstrate that from a small region of motor cortex $(<$ $5 \mathrm{~mm}$ ) it is possible, with a high level of significance, to discern cortical changes associated with contra- and ipsilateral distal arm movements.

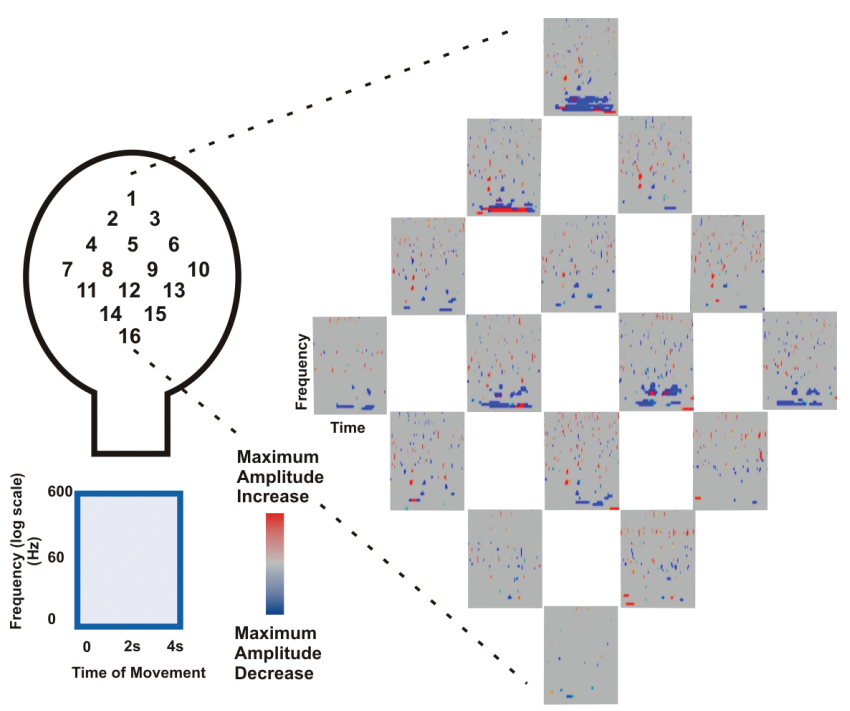

FIG. 2. Separating contralateral and ipsilateral movements. The figure demonstrates the averaged time frequency amplitude difference that was statistically different between ipsi- and contralateral movements. The time frequency plots are shown for each microelectrode (numbered on the left and shown on the right). The most prominent differences were more often found in lower-frequency bands $(<40 \mathrm{~Hz})$ and in Electrodes 1, 2, 8, 9, and 10. 


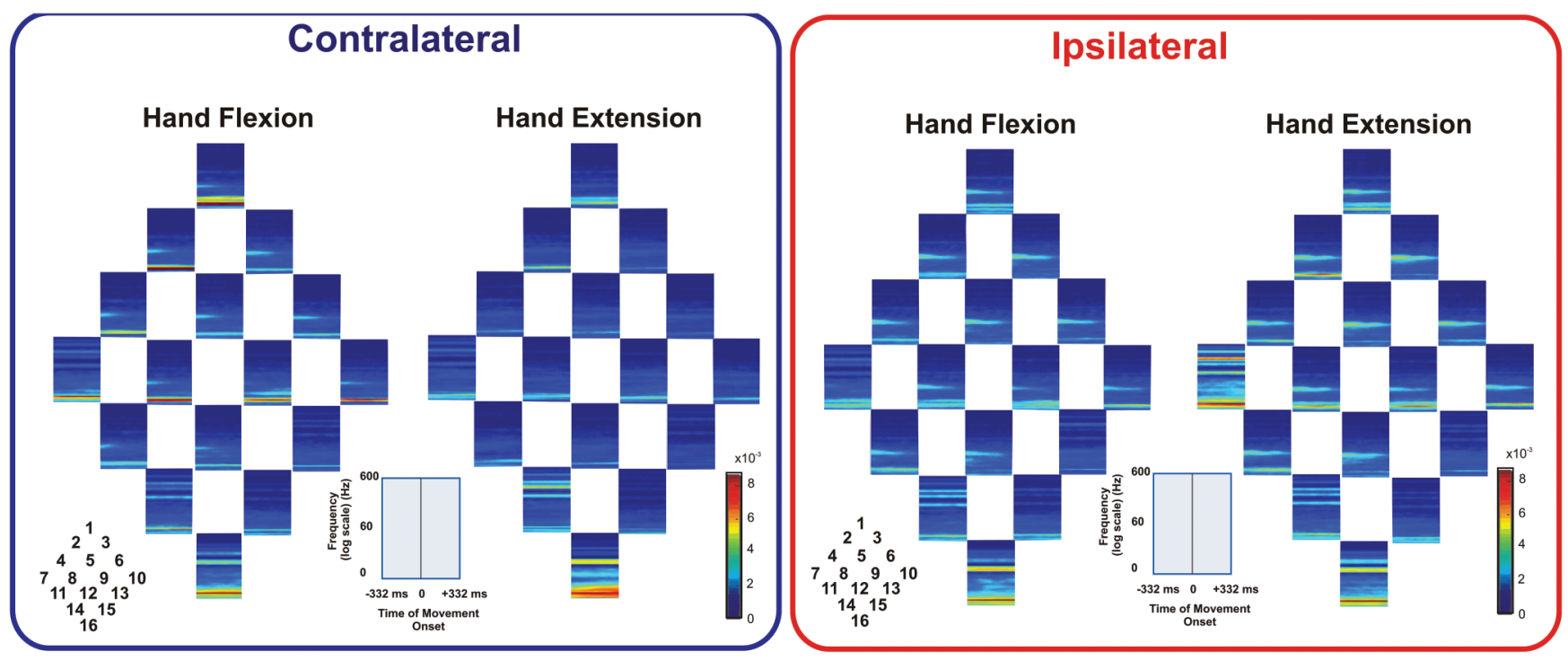

FIG. 3. Separating wrist flexion and extension from the contralateral limb. The figure demonstrates the averaged mutual information between the frequency spectra of each individual microelectrode and the EMG recording from the dorsal and ventral aspect of the forearm. Dorsal EMG is associated with wrist extension and ventral EMG changes are associated with wrist flexion. Left: The time scale shows the shared mutual information over a range of $332 \mathrm{msec}(\mathrm{ms})$ before and after the onset of movement. For contralateral wrist movements, there is an increased contribution from $20-30-\mathrm{Hz}$ signal change prior to the onset of movement, which occurs diffusely in the majority of electrodes with wrist flexion that is not present with wrist extension. Additionally, Electrodes 1, 2, and 7-10 show a very low-frequency predominance associated with flexion that is not present with extension. Wrist extension on the other hand shows a notable broadband frequency information content from 10 to $50 \mathrm{~Hz}$ in Electrode 16 not present with flexion. These findings indicate that there are qualitative differences in cortical activity associated with flexion and extension in the contralateral (left) arm. Right: The time scale shows the shared mutual information over a range of $332 \mathrm{msec}$ before and after the onset of movement. Ipsilateral wrist flexion and extension movements are more similar than that seen with contralateral wrist movements. There is an anatomically diffuse frequency component that largely precedes the onset of movement in a frequency range of $30-50 \mathrm{~Hz}$. There is, however, a notable difference in Electrode 7 demonstrating higher-frequency information content associated with wrist extension not seen in flexion. These findings indicate that there are qualitative differences in cortical activity associated with flexion and extension in the ipsilateral (right) arm.

\section{Separating Wrist Flexion and Extension From Each Arm}

To separate wrist flexion and extension for a given hand, the probabilistic method of mutual information was used. Distinct from a right/left paradigm of move/do not move, one cannot flex without extending in a blocked format. In other words, one cannot continually extend during a period of time and then continually flex during a given period of time. If one extends one's wrist, even to return it to neutral, it requires one to flex the wrist to some degree. To address this, EMG was continually recorded from the dorsal and ventral aspect of the forearm, and the mutual information between the ECoG signal of the microarray and the EMG signals of the forearm electrodes was determined. Thus, ECoG signals showing increased mutual information with dorsal forearm EMG reveals cortical activity associated with a wrist extension, whereas ECoG signals showing mutual information with ventral EMG activity reveals cortical activity associated with wrist flexion.

The mutual information was evaluated over a range from $332 \mathrm{msec}$ before and after the initiation of an onset of movement identified with EMG across $0-600 \mathrm{~Hz}$ and averaged across all trials. The time series was then analyzed to assess whether there were changes that were distinct to a given movement for a given limb. For contralat- eral wrist movements, as shown in Fig. 3 left, there was an increased contribution from $20-$ to $30-\mathrm{Hz}$ signal change prior to the onset of movement, which occurred diffusely in the majority of electrodes with wrist flexion but was not present with wrist extension. Additionally, Electrodes 1, 2 , and 7-10 exhibited a very low frequency predominance with flexion that was not present with extension. Wrist extension on the other hand showed a notable broadband frequency information content from $10-50 \mathrm{~Hz}$ in Electrode 16, which was not present with flexion. Figure 3 right shows similar data for ipsilateral wrist movements. Ipsilateral flexion and extension movements were more similar than contralateral wrist movements. There was an anatomically diffuse frequency component that largely preceded the onset of movement in a frequency range of $30-50 \mathrm{~Hz}$. There was, however, a notable difference in Electrode 7, demonstrating higher-frequency information content associated with wrist extension not seen in flexion. Taken together, these findings demonstrate that there are qualitative differences in cortical activity associated with flexion and extension in both the contra- and ipsilateral arms.

\section{Distinguishing Spectral and Anatomical Patterns of Flexion and Extension Across Both Arms}

To assess whether distinguishable anatomical and 
E. C. Leuthardt et al.

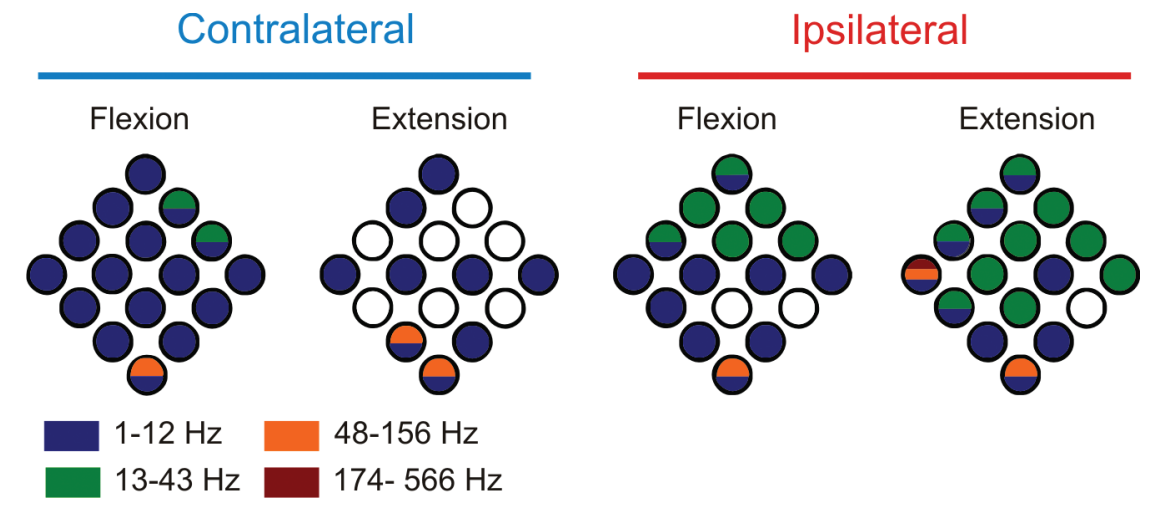

FIG. 4. Distinguishing spectral and anatomical patterns of flexion and extension across both arms. The figure shows the patterns of mutual information in terms of anatomical location and frequency on the microarray. The spectral information was separated into 4 separate frequency bands: low (1-12 Hz), intermediate (13-43 Hz), high (48-152 Hz), and very high (174-566 $\mathrm{Hz}$ ). Each condition has a distinct anatomical pattern of cortical activity in terms of which electrode participates in encoding either ipsi- or contralateral wrist flexion or extension. Additionally, there is a different spectral representation between each of the 4 conditions. Contralateral movements seem to be more predominantly represented by the low-frequency band $(1-12 \mathrm{~Hz})$, whereas ipsilateral movements are more highly represented by the intermediate frequency band $(13-43 \mathrm{~Hz})$. Each had focal high-frequency changes that were anatomically distinct to the given condition.

spectral cortical patterns are present to potentially distinguish between each of the 4 movement conditions (that is, contralateral flexion, contralateral extension, ipsilateral flexion, and ipsilateral extension) the time series data for individual movements was then taken together and "thresholded" relative to the maximum mutual information shared between the recorded ECoG signal and the EMG signals. The spectral information was separated into 4 separate frequency bands-low $(1-12 \mathrm{~Hz})$, intermediate $(13-43 \mathrm{~Hz})$, high $(48-152 \mathrm{~Hz})$, and very high $(174-566 \mathrm{~Hz})$. An electrode was deemed to be "positive" if $>15 \%$ the maximum mutual information between the ECoG signal and EMG signal was present. Figure 4 shows the patterns of mutual information in terms of anatomical location and frequency on the microarray. Each condition has a distinct anatomical pattern of cortical activity in terms of which electrode participated in encoding either ipsilateral or contralateral wrist flexion or extension. Additionally, there is a different spectral representation between each of the 4 conditions. Contralateral movements seemed to be more predominantly represented by the low-frequency band $(1-12 \mathrm{~Hz})$, whereas ipsilateral movements are more highly represented by the intermediate-frequency band $(13-43 \mathrm{~Hz})$. Each had focal high-frequency changes that were anatomically distinct to the given condition.

\section{Discussion}

The results of this study demonstrated that ECoG recordings obtained in small regions $(<5 \mathrm{~mm})$ of primary motor cortex can yield substantial information about bilateral distal limb movements. We found that there are statistically significant differences between contraand ipsilateral wrist movements represented by distinct frequency bands (frequencies $<40 \mathrm{~Hz}$ ) and anatomical patterns of activation. Using nonlinear probabilistic methods, wrist flexion and extension were further subsegmented based on the mutual information shared between the ECoG signal and the EMG activity recorded from the forearm. There were anatomically and spectrally distinct patterns of mutual information between each movement condition. In summary, these findings support the possibility that a small ECoG electrode array could provide sufficient information about motor intentions for potential neuroprosthetic operation in the future.

The findings in this study are consistent with human cortical physiology studies in which larger-scale recordings of EEG and macroscale (1-cm electrode spacing) ECoG have been used. The classic cortical physiology associated with contralateral limb and hand movements has demonstrated specific amplitude changes at various frequency ranges of oscillating brain activity. These frequencies have been categorized into 3 main functional groups as they relate to sensorimotor cortex. Sensorimotor rhythms comprise the mu band $(8-12 \mathrm{~Hz})$, the beta band $(18-26 \mathrm{~Hz})$, and the gamma band $(>40 \mathrm{~Hz}) .^{13,24,36} \mathrm{Mu}$ and beta bands are thought to represent synaptic potentials produced by thalamocortical circuits, and they change in amplitude in association with actual or imagined movements. ${ }^{10,18,23,26}$ The higher frequencies in the gamma band are thought to be produced by smaller cortical assemblies and also have been found to change in amplitude relative to active or imagined motor movements. ${ }^{6,17,19}$ In general, the lower-frequency bands (mu and beta) tend to exhibit broader cortical regions of amplitude decrease, while the higher gamma band tends to show a more focal increase in amplitude when the region of cortex associated with the motor action becomes active. ${ }^{21-23}$ On this much smaller scale, the lower-frequency changes tended to be more diffuse while high-frequency changes were much more cortically constrained to 1 to 2 microelectrodes. More recently, when contralateral hand movements were compared with ipsilateral hand movements, there was found to be a distinct and separable cortical physiology associated with same-side movements. The cortical changes associated with ipsilateral hand movements were found to occur in lower-frequency spectra (average $37.5 \mathrm{~Hz}$ ), at distinct 


\section{Microscale ECoG recording in the human motor cortex}

anatomical locations (most notably in the premotor cortex), and earlier (by $160 \mathrm{msec}$ ) than changes associated with contralateral hand movements. These observations were found to be consistent with a motor planning role of ipsilateral cortex in motor movements. ${ }^{34}$ Consistent with these findings, ipsilateral wrist movements were found to have the most mutual information in a frequency range of $13-43 \mathrm{~Hz}$. Additionally, this range appeared to largely precede the onset of movement.

The small scale with which motor information was acquired in this study has important surgical implications for BCI applications in the future. To date, ECoG recording has primarily occurred through electrode arrays made specifically for epilepsy monitoring. The role of these grid electrodes is to achieve broad cortical coverage for the localization of a seizure focus and as a result require a fairly large craniotomy for their placement. The demonstration that separable motor intentions can be acquired from a site in primary motor cortex that is $<1 \mathrm{~cm}$ indicates that the practical array for neuroprosthetic applications may only require a $1-\mathrm{cm}$ bur hole. This would significantly reduce the risk of the surgical procedure for placement of the BCI construct. Additionally, previous studies have shown that epidurally acquired ECoGs signal can be used for brain-derived device control. ${ }^{15}$ If the microscale nature of these signals is preserved above and below the dura mater, a construct that requires a bur hole and an epidural array could be even less risky. The difference in signal fidelity, however, will need to be explicitly studied. The significant risk reduction could improve the risk-benefit consideration for implantation in either medically fragile patients (such as those with amyotrophic lateral sclerosis) or in patients in whom the construct would be placed over normal brain (such as those with spinal cord injury or an unaffected hemisphere after unilateral stroke). This ultimately could hasten the adoption of the BCI technology across a wider patient population.

Although exciting, there are some important caveats of which to take note. This study demonstrates that there is indeed cortical activity present on a millimeter scale that reveals substantial information about various motor movements. Although probabilistic methods, such as mutual information, can distinguish motor intentions, further studies will be required for real-time signal analysis and testing to determine how effective these features are for the application of user control. This will require that the various signals be independent ( 1 cortical change associated with 1 motor intention is not easily confused with a cortical change associated with a different motor intention) and controllable by the individual (the patient can use a cognitive task to reliably control an external device). What the anatomical resolution means for independent control features remains to be determined. Also, when a very small array is considered for a BCI, where it will be placed becomes more critical. Understanding the relationship between electrocortical activation and noninvasive methods of localizing these changes will also be important. The bur hole and array will have to be placed in a region sufficiently close to the region of interest such that it will provide the necessary independent signals. There has been preliminary evidence that functional MR imaging is useful for identifying regions of cortical activation that, when investigated with $\mathrm{ECoG}$, will provide signals for BCI device operation. Further studies will be required to define more fully the reliability of this technique across multiple different cognitive paradigms. ${ }^{25}$ Additionally, work is also being done to create implantable microarrays that can be used for prolonged recording and signal transmission. ${ }^{11,12}$ To complement these emerging efforts, this study provides an important first step in showing that small cortical recording may provide the necessary signals for a minimally invasive ECoG BCI. The next steps will require the application of these signals for effective device control and a reliable method for noninvasive localization of where in cortex these signals are coming.

\section{Conclusions}

In summary, we have recorded from microelectrode array over primary motor cortex in a humans. The cortical information was sufficient to distinguish between contra- and ipsilateral wrist flexion and extension movements. This provides exciting preliminary evidence that a BCI implant may only require a small region of cortical coverage to provide sufficient information about motor intentions for brain-derived device control.

\section{Disclosure}

Dr. Leuthardt owns stock in the company Neurolutions.

Dr. Leuthardt has received grant funding from the following: James S. McDonnell Foundation; Higher Brain Function; Department of Defense (No. W911NF-07-1-0415 and W911NF-08-1-0216); National Institutes of Health (No. R01-EB000856-06); and the Children's Discovery Institute.

\section{References}

1. Bjornsson CS, Oh SJ, Al-Kofahi YA, Lim YJ, Smith KL, Turner JN, et al: Effects of insertion conditions on tissue strain and vascular damage during neuroprosthetic device insertion. J Neural Eng 3:196-207, 2006

2. Blakely T, Miller KJ, Rao RP, Holmes MD, Ojemann JG: Localization and classification of phonemes using high spatial resolution electrocorticography $(\mathrm{ECoG})$ grids. Conf Proc IEEE Eng Med Biol Soc 2008:4964-4967, 2008

3. Boulton AA, Baker GB, Vanderwolf CH: Neurophysiological Techniques II: Applications to Neural Systems. Totowa. New Jersey: Humana Press, 1990

4. Cover TM, Thomas JA: Elements of Information Theory. New York: John Wiley \& Sons, 1991

5. Cox RW: AFNI: software for analysis and visualization of functional magnetic resonance neuroimages. Comput Biomed Res 29:162-173, 1996

6. Crone NE, Miglioretti DL, Gordon B, Lesser RP: Functional mapping of human sensorimotor cortex with electrocorticographic spectral analysis. II. Event-related synchronization in the gamma band. Brain 121:2301-2315, 1998

7. Dale AM, Fischl B, Sereno MI: Cortical surface-based analysis. I. Segmentation and surface reconstruction. Neuroimage 9:179-194, 1999

8. Freeman WJ, Holmes MD, Burke BC, Vanhatalo S: Spatial spectra of scalp EEG and EMG from awake humans. Clin Neurophysiol 114:1053-1068, 2003

9. Hochberg LR, Serruya MD, Friehs GM, Mukand JA, Saleh 
M, Caplan AH, et al: Neuronal ensemble control of prosthetic devices by a human with tetraplegia. Nature 442:164-171, 2006

10. Huggins JE, Levine SP: BeMent SL, Kushwaha RK, Schuh LA, Passaro EA, et al: Detection of event-related potentials for development of a direct brain interface. J Clin Neurophysiol 16:448-455, 1999

11. Kim J, Wilson JA, Williams JC: A cortical recording platform utilizing microECoG electrode arrays. Conf Proc IEEE Eng Med Biol Soc 2007: 5353-5357, 2007

12. Kitzmiller J, Beversdorf D, Hansford D: Fabrication and testing of microelectrodes for small-field cortical surface recordings. Biomed Microdevices 8:81-85, 2006

13. Kostov A, Polak M: Parallel man-machine training in development of EEG-based cursor control. IEEE Trans Rehabil Eng 8:203-205, 2000

14. Kubler A, Nijboer F, Mellinger J, Vaughan TM, Pawelzik $\mathrm{H}$, Schalk G, et al: Patients with ALS can use sensorimotor rhythms to operate a brain-computer interface. Neurology 64:1775-1777, 2005

15. Leuthardt EC, Miller KJ, Schalk G, Rao RP, Ojemann JG: Electrocorticography-based brain computer interface-the Seattle experience. IEEE Trans Neural Syst Rehabil Eng 14:194-198, 2006

16. Leuthardt EC, Schalk G, Moran D, Ojemann JG: The emerging world of motor neuroprosthetics: a neurosurgical perspective. Neurosurgery 59:1-14, 2006

17. Leuthardt EC, Schalk G, Wolpaw JR, Ojemann JG, Moran DW: A brain-computer interface using electrocorticographic signals in humans. J Neural Eng 1:63-71, 2004

18. Levine SP, Huggins JE: BeMent SL, Kushwaha RK, Schuh LA, Passaro EA, et al: Identification of electrocorticogram patterns as the basis for a direct brain interface. J Clin Neurophysiol 16:439-447, 1999

19. Lopes da Silva FH, Pfurtscheller G (eds): Event-Related Desynchronization. Handbook of Electroencephalography and Clinical Neurophysiology. Amsterdam: Elsevier, 1999

20. Miller KJ, Leuthardt EC, Schalk G, Rao RP, Anderson NR, Moran DW, et al: Spectral changes in cortical surface potentials during motor movement. J Neurosci 27:2424-2432, 2007

21. Pfurtscheller G: Event-Related Desynchronization (ERD) and Event Related Synchronization (ERS). Baltimore: Williams and Wilkins, 1999

22. Pfurtscheller G, Berghold A: Patterns of cortical activation during planning of voluntary movement. Electroencephalogr Clin Neurophysiol 72:250-258, 1989

23. Pfurtscheller G, Graimann B, Huggins JE, Levine SP, Schuh LA: Spatiotemporal patterns of beta desynchronization and gamma synchronization in corticographic data during selfpaced movement. Clin Neurophysiol 114:1226-1236, 2003

24. Pfurtscheller G, Neuper C, Guger C, Harkam W, Ramoser H, Schlogl A, et al: Current trends in Graz brain-computer interface (BCI) research. IEEE Trans Rehabil Eng 8:216-219, 2000
25. Ramsey NF, van de Heuvel MP, Kho KH, Leijten FS: Towards human BCI applications based on cognitive brain systems: an investigation of neural signals recorded from the dorsolateral prefrontal cortex. IEEE Trans Neural Syst Rehabil Eng 14:214-217, 2006

26. Rohde MM: BeMent SL, Huggins JE, Levine SP, Kushwaha RK, Schuh LA: Quality estimation of subdurally recorded, event-related potentials based on signal-to-noise ratio. IEEE Trans Biomed Eng 49:31-40, 2002

27. Saad ZS, Glen DR, Chen G, Beauchamp MS, Desai R, Cox RW: A new method for improving functional-to-structural MRI alignment using local Pearson correlation. Neuroimage 44:839-848, 2009

28. Schalk G, Kubanek J, Miller KJ, Anderson NR, Leuthardt EC, Ojemann JG, et al: Decoding two-dimensional movement trajectories using electrocorticographic signals in humans. J Neural Eng 4:264-275, 2007

29. Schalk G, McFarland DJ, Hinterberger T, Birbaumer N, Wolpaw JR: BCI2000: a general-purpose brain-computer interface (BCI) system. IEEE Trans Biomed Eng 51:1034-1043, 2004

30. Schalk G, Miller KJ, Anderson NR, Wilson JA, Smyth MD, Ojemann JG, et al: Two-dimensional movement control using electrocorticographic signals in humans. J Neural Eng 5:75-84, 2008

31. Srinivasan R, Nunez PL, Silberstein RB: Spatial filtering and neocortical dynamics: estimates of EEG coherence. IEEE Trans Biomed Eng 45:814-826, 1998

32. Szarowski DH, Andersen MD, Retterer S, Spence AJ, Isaacson M, Craighead HG, et al: Brain responses to micro-machined silicon devices. Brain Res 983:23-35, 2003

33. Vetter RJ, Williams JC, Hetke JF, Nunamaker EA, Kipke DR: Chronic neural recording using silicon-substrate microelectrode arrays implanted in cerebral cortex. IEEE Trans Biomed Eng 51:896-904, 2004

34. Wisneski KJ, Anderson N, Schalk G, Smyth M, Moran D, Leuthardt EC: Unique cortical physiology associated with ipsilateral hand movements and neuroprosthetic implications. Stroke 39:3351-3359, 2008

35. Wolpaw JR, McFarland DJ: Control of a two-dimensional movement signal by a noninvasive brain-computer interface in humans. Proc Natl Acad Sci U S A 101:17849-17854, 2004

36. Wolpaw JR, McFarland DJ, Neat GW, Forneris CA: An EEGbased brain-computer interface for cursor control. Electroencephalogr Clin Neurophysiol 78:252-259, 1991

Manuscript submitted March 15, 2009.

Accepted April 30, 2009.

Address correspondence to: Eric C. Leuthardt, M.D., Department of Neurological Surgery, Center for Innovation in Neuroscience and Technology, Washington University School of Medicine, 660 South Euclid Avenue, Campus Box 8057, St. Louis, Missouri 63110. email: leuthardte@nsurg.wustl.edu. 